Research Article

\title{
An Ensemble Model of Wind Speed Forecasting Based on Variational Mode Decomposition and Bare-Bones Fireworks Algorithm
}

\author{
Jicheng Quan (iD) and Li Shang \\ Department of Computer Science and Technology, Tongji University, No. 4800 Caoan Rd, Jiading District, \\ Shanghai 201804, China \\ Correspondence should be addressed to Jicheng Quan; 1510485@tongji.edu.cn
}

Received 30 November 2020; Revised 17 February 2021; Accepted 23 February 2021; Published 8 March 2021

Academic Editor: Petr H jek

Copyright ( $) 2021$ Jicheng Quan and Li Shang. This is an open access article distributed under the Creative Commons Attribution License, which permits unrestricted use, distribution, and reproduction in any medium, provided the original work is properly cited.

\begin{abstract}
Wind energy is one of the fastest growing renewable energy sources. Wind speed forecasting is essential to enhance the utilization of wind energy. Various prediction models have been developed to improve the prediction accuracy of wind speed. However, wind speed time series has nonlinearity, fluctuation, and intermittence, which makes the prediction difficult. Existing prediction models ignore data decomposition and feature reduction and suffer from the deficiency of individual models. This paper proposes a novel ensemble prediction model, which integrates data preprocessing, feature selection, parameter optimization, three intelligent prediction models, and an ensemble strategy. To improve prediction performance, a highly efficient optimization algorithm is applied to determine the individual models' optimal parameters. Furthermore, partial least square regression is used to calculate combination weight. Additionally, two 10 min datasets from the National Renewable Energy Laboratory (NREL) are employed for one-step-ahead prediction. Among the involved models, the proposed model can obtain the best prediction accuracy. The experimental results indicate that the mean absolute percent errors of the proposed model are $7.97 \%$ and $9.99 \%$, which are lower than the comparison methods. Pearson's test reveals that the proposed approach can have the strongest association between the actual data and the prediction results.
\end{abstract}

\section{Introduction}

1.1. Motivation. With the development of economy and society, the contradiction between energy demand and energy supply is escalated. As fossil energy sources are depleted, the energy transition has become an issue of great concern [1]. Wind power is an effective alternative to fossil energy, cheap, environmentally friendly, and widely distributed. It is one of the world's fastest growing renewable energy sources. According to statistics, by 2020, wind power's global cumulative installed capacity has reached 703 GW. However, wind speed has discontinuity and randomness. The randomness of wind power harms the smooth operation of the power system. Effective wind speed prediction can enhance the safety of power system operation and improve wind energy efficiency. However, wind speed is characterized by its low density, instability, and regional variation. These factors make it very difficult to predict wind speed accurately. In the past two decades, researchers have developed many wind speed prediction methods to improve the prediction accuracy. According to mathematical principles, these models are divided into four categories, namely, physical models, statistical models, artificial intelligence models, and hybrid models [2]. The advantages and drawbacks of the above methods are discussed in Section 1.2.

1.2. Literature Review. The physical approach utilizes meteorological data and atmospheric dynamics to simulate wind speed. In this field, numerical weather prediction (NWP) models have been exploited for medium-term wind speed forecasting; for example, Zhao et al. [3] established an 
adaptive structured error correction model to improve NWP predictions. The results indicated that error correction could effectively enhance forecast accuracy. Olson et al. [4] adopted field observations to reduce NWP forecast errors. The simulations could reduce the mean absolute errors by 5\%-20\%. Liu et al. [5] used kernel density estimation to process wind speed prediction deviation. These studies show that error correction is suitable for physical models, and the physical method exceeds persistence methods.

The statistical approach uses historical data to structure the linear relationship. The typical strategies are time-series analysis methods and regression models. In previous studies, Zhang et al. [6] proposed an autoregressive and moving average (ARMA) model to predict the linear part of wind speed. The results showed that the ARMA model could structure the actual characteristics of wind speed. Karaku et al. [7] presented a polynomial autoregressive (PAR) model for one-day-ahead wind speed prediction, which outperformed the ARMA model. Qin et al. [8] developed a smooth transition periodic autoregressive (STPAR) model to predict the six-hourly wind speeds. In the study, they employed an artificial neural network to correct the prediction errors of the STPAR model. The above studies indicate that the statistical approach is practical for wind speed prediction. It is more efficient than the physical method; however, it has shortcomings in resolving nonlinear problems.

The artificial intelligence (AI) approach is beneficial to structure the nonlinear relationship. The usual methods include artificial neural networks (ANNs), extreme learning machines (ELMs), and support vector machines (SVMs); for example, Kadhem et al. [9] developed an ANN model to exploit seasonal weather variation's dependencies in wind speed prediction. Wang et al. [10] proposed an echo state network (ESN) and showed that the ESN model achieved excellent accuracy and stability. Tian et al. [11] proposed an error minimized extreme learning machine. The results indicated that ELMs were more efficient than ANNs. Zhang et al. [12] proposed an online sequential extreme learning machine (OSELM) model for wind speed prediction. The results revealed that OSELM could exceed offline ELM models. Besides ANNs and ELMs, SVMs are the other popular models in wind speed prediction [13]; for example, Fu et al. [14] proposed an improved chicken algorithm optimized SVM. Ren et al. [15] offered the least squares support vector regression (LSSVR) for wind speed prediction and proved that LSSVR was superior to ARMA and ANN. However, these AI models still have drawbacks. The ANNs have difficulties in specifying network structures. ELMs have underfitting issues in small datasets. SVMs have inefficiency in large datasets. To overcome these defects, the hybrid approach is proposed. In general, such a process comprises data preprocessing, feature selection, parameter optimization, and separate AI models. These methods are reviewed in the following sections.

Data preprocessing can reduce prediction complexity $[16,17]$. By referring to previous studies, signal decomposition methods have been used for prediction, including wavelet packet decomposition (WPD), empirical mode decomposition (EMD), ensemble empirical mode decomposition (EEMD), and variational mode decomposition (VMD); for example, Ye et al. [18] adopted WPD to break down the wind speed series into wavelet spaces and removed the data noise. The correctness of the method was proved in a wind plant in China. Mi et al. [19] used EMD to extract the wind fluctuation features. Yang and Yang [20] used EEMD to decompose the wind time series into subsequences and established a Bayesian ridge regression model per each subsequence. The model outperformed the single AI models. Recently, EEMD has been integrated into deep learning frameworks. Santhosh et al. [21] combined EEMD and deep Boltzmann machines (DBMs) and achieved accurate results. Huang et al. [22] established a long short-term memory (LSTM) neural network per each EEMD subsequence. This study proved that EEMD could enhance the prediction accuracy of LSTM. However, the above algorithms still have deficiencies. WPD has a flaw in processing low-frequency wind subseries. EMD and EEMD have the mode mixing issue. In this paper, $\mathrm{VMD}$ is used to resolve the above limitations. The validity of VMD has been demonstrated successfully in wind speed prediction [23]; for instance, Wang et al. [24] proposed a hybrid model on VMD and SVM. The results indicated that VMD was superior to EEMD. Similarly, Wu and Lin [25] developed a combined model on VMD and LSSVR. They optimized LSSVR by the bat algorithm (BA). The proposed model surpassed the LSSVM and BA-LSSVM models.

Feature selection can decrease the computation of prediction models. They can be divided into two categories: wrapper and filter. The representative filter methods are partial autocorrelation function (PACF) and mutual information (MI); for example, Sun et al. [26] employed PACF to eliminate illusive components for wind decomposition subsignals. Memarzadeh and Keynia [27] used MI to select the optimal features for LSTM and showed its effectiveness. Huang et al. [28] proposed a hybrid feature selection method. First, conditional mutual information (CMI) was applied to reduce feature redundancy. Then, the forward feature selection approach was performed to obtain the final feature subsets. In recent years, the binary genetic algorithm (BGA) has been proposed for feature selection, and it can produce better prediction accuracy than the wrapper approaches. Therefore, in this paper, we adopt BGA for wind speed prediction. As a binary variant of the genetic algorithm, BGA can enhance prediction efficiency and accuracy. For instance, Semero et al. [29] employed BGA to select power load patterns and successfully eliminated redundant features. Liu et al. [30] adopted BGA in wind speed forecasting and demonstrated that BGA was sufficient for multistep prediction.

Optimization algorithms can improve the performance of prediction models on the training datasets. The representative algorithms are the genetic algorithm (GA), particle swarm optimization (PSO), differential evolution (DE), and artificial bee colony (ABC) algorithm. For instance, Liu et al. [31] used GA to adjust the parameters of a multilayer perceptron (MLP) neural network. Bezerra, Leao, and Braga [32] modified PSO with ageing and weakening factors and 
verified the algorithm on a focused time-delay neural network. The results showed that the training time was notably reduced, and the prediction errors were decreased. $\mathrm{Hu}$ and Chen [33] employed DE to optimize the structure of LSTM. Tian et al. [11] adopted $\mathrm{ABC}$ to determine the optimal parameters of ELM. Simulation results indicated that the training time of ELM was reduced. Although the above algorithms can improve the prediction performance, they have inherent defects. For instance, GA has a slow convergence speed, and PSO is sensitive to parameter tuning. Recently, a novel optimization algorithm, namely, the barebones fireworks algorithm (BBFWA), has been proposed. It has fewer parameters and lower computation complexity than GA, PSO, and DE. Athar et al. [34] applied BBFWA to power plant site selection problem. The study indicated that BBFWA was simple, fast, and accurate for site selection. Lei et al. [35] used BBFWA to optimize LSSVR for power load prediction. The results indicated that BBFWA was more efficient than cross-validation. Therefore, we employ BBFWA to fine-tune individual models. To our best knowledge, in this paper, BBFWA is being used in wind speed prediction for the first time.

However, each hybrid model has its inherent deficiencies, and the ensemble approach can take the advantages of prediction models while improving their shortcomings [36]. In an ensemble model, several individual models perform the forecasting, respectively, and then an ensemble algorithm is employed to determine the weight coefficients for model combination. Xiao et al. [37] developed the no negative constraint theory (NNCT), which suggested that the optimal weights shall be in the range of $[-2,2]$. Consequently, metaheuristic algorithms were utilized to optimize weight coefficients; for example, Yang and Wang [38] used the water cycle algorithm to calculate the weight coefficients. The simulations indicated that the combined model was superior to individual models. Niu and Wang [39] employed the grasshopper algorithm to ensemble four neural network models which achieved stable prediction accuracy. Luo et al. [40] adopted the multiobjective cuckoo search algorithm to decide the weight coefficients. However, when several models resolve the same prediction problem, their outputs may be highly correlated, and the metaheuristic algorithms may not effectively eliminate such correlation. To address this issue, we use partial least squares regression (PLSR) to estimate the weight coefficients. By referring to previous studies, Jiang and Li [41] adopted PLSR to ensemble several neural networks for wind speed prediction. The results indicated that PLSR could reduce the chance of overfitting and improve individual models' accuracy and reliability.

1.3. Research Contribution. Over the years, researchers have proposed plenty of wind speed prediction models. The representative models are summarized in Table 1 . It can be seen that some prediction models rely on physical simulations or historical data. However, due to wind speed's randomness, such methods cannot achieve stable and accurate prediction results. The AI models often have inefficiency or lack of online learning capability. The filter approaches usually produce less prediction accuracy than the wrapper approaches. Swarm intelligence algorithms may have a slow convergence speed or be sensitive to parameter tuning. The optimization-based ensemble strategies may not sufficiently eliminate the correlations for individual models. This paper presents a novel ensemble model based on data preprocessing, state-of-the-art optimization, and NNCT theory to repair the above issues. The proposed model combines VMD, BGA, BBFWA, LSSVR, ESN, OSELM, and PLSR. The contributions of the paper are as follows:

(1) A novel ensemble model is proposed for wind speed prediction. Compared with hybrid models, the proposed model overcomes the individual models' inherent limitations and achieves better accuracy. Besides, PLSR is employed to estimate the weight coefficients. The proposed weight estimation method has better efficiency and robustness than heuristic optimization-based methods.

(2) To our best knowledge, it is the first time BBFWA has been applied to wind speed forecasting. Compared with traditional optimization algorithms, BBFWA has a more straightforward structure and lower computational complexity. Our study indicates that BBFWA can effectively improve the performance of the models.

(3) Signal decomposition and feature selection are combined to generate optimal features. VMD is adopted to decompose the original wind series. Compared with EMD and EEMD, VMD can generate sublayers with precise physical meanings. Besides, VMD can avoid the mode mixing effect. BGA is used to select the optimal features from the sublayers. Compared with correlation-based approaches, BGA can obtain more compelling features.

(4) The proposed model has efficiency and the capability of online learning. In the proposed approach, LSSVR, OSELM, and ESN are adopted as the basic models. LSSVR has a simpler mathematical structure than SVR. OSELM has a unique online learning capability. ESN has better generalization ability than ANNs. The proposed method integrates the above three models to complete the forecasting. Consequently, our proposed model achieves both efficiency and the capability of online learning.

(5) Several experiments are conducted to prove the effectiveness of the proposed model. In this study, eleven models are implemented for comparison. The experimental results indicate that the proposed model is superior to the other involved models.

The remaining part of the paper proceeds as follows: Section 2 introduces the related methods and proposed hybrid model, Sections 3 and 4 present the experimental results and discussion, and Section 5 draws the conclusions.

\section{Proposed Approach}

In this section, the proposed approach's details are presented, including the signal decomposition, feature 
TABLE 1: Summary of reviewed forecasting models.

\begin{tabular}{|c|c|c|c|}
\hline Wind speed forecasting models & Advantages & Disadvantages Reference papers & \\
\hline \multicolumn{4}{|c|}{ Physical methods } \\
\hline \multicolumn{4}{|c|}{ Statistical methods } \\
\hline Time series models & Excellence in modelling linear data & $\begin{array}{c}\text { Limitation to process nonlinear } \\
\text { data }\end{array}$ & {$[6-8]$} \\
\hline \multicolumn{4}{|c|}{ Artificial intelligence methods } \\
\hline ANN & Good performance in modelling nonlinear problems & $\begin{array}{l}\text { Difficulty in determining the } \\
\text { network structure }\end{array}$ & {$[9,10]$} \\
\hline ELM & High learning efficiency & $\begin{array}{l}\text { Random initialization of the input } \\
\text { weight of the network }\end{array}$ & {$[11,12]$} \\
\hline SVM & Kernel tricks and solid mathematical theory & $\begin{array}{c}\text { Inefficiency in handling large- } \\
\text { scale dataset }\end{array}$ & [13-15] \\
\hline \multicolumn{4}{|c|}{ Signal preprocessing methods } \\
\hline WPD & Good properties in the frequency domain & $\begin{array}{l}\text { Difficulty in determining the } \\
\text { decomposition level }\end{array}$ & {$[18]$} \\
\hline EMD & Adaptive decomposition & $\begin{array}{l}\text { Endpoint effect and mode mixing } \\
\text { issue }\end{array}$ & [19] \\
\hline EEMD & Adaptive decomposition & $\begin{array}{c}\text { Noise issue in the reconstructed } \\
\text { signal }\end{array}$ & {$[20-22,42]$} \\
\hline VMD & Band-limited intrinsic mode functions & Nonrecursive decomposition & [23-25] \\
\hline \multicolumn{4}{|c|}{ Feature selection methods } \\
\hline PACF & Based on probability statistics & $\begin{array}{l}\text { Inefficiency in handling nonlinear } \\
\text { relations }\end{array}$ & {$[26]$} \\
\hline Mutual information & Based on information theory & Lower prediction accuracy & {$[27,28]$} \\
\hline BGA & Good applicability & Massive calculation & {$[29,30]$} \\
\hline $\begin{array}{l}\text { Metaheuristic optimization } \\
\text { Regression }\end{array}$ & $\begin{array}{l}\text { Ensemble methods } \\
\text { Strong reliability } \\
\text { Efficiency }\end{array}$ & $\begin{array}{l}\text { Massive calculation } \\
\text { Overfitting issue }\end{array}$ & $\begin{array}{c}{[37-40,43]} \\
{[41]}\end{array}$ \\
\hline
\end{tabular}

selection, individual models, the optimization algorithm, and the weight coefficient determination method.

\subsection{Variational Mode Decomposition. The VMD algorithm} is developed to overcome the limitations of EMD [44]. It can decompose an original signal $x(t)$ into intrinsic mode functions (IMFs). The literature presented significant advantages in time series forecasting [45] and fault diagnosis [46]. The core principle of VMD is to realize the IMFs by resolving the following constrained optimization problem [47]:

$$
\min _{\left\{u_{k}\right\},\left\{\omega_{k}\right\}}\left\{\sum_{k} \partial_{t}\left[\left(\delta(t)+\frac{j}{\pi t}\right) * u_{k}(t)\right] e^{-j \omega_{k} t^{2}}\right\}
$$

subject to

$$
\sum_{k=1}^{K} u_{k}(t)=x(t)
$$

where $\left\{u_{k}\right\}=\left\{u_{1}, u_{2}, \ldots, u_{K}\right\}$ denotes the IMFs, $\left\{\omega_{k}\right\}=\left\{\omega_{1}, \omega_{2}, \ldots, \omega_{K}\right\}$ is a central frequency of each IMF in the Fourier frequency domain, and $\delta(t)$ represents a Dirac function. The constraint conditions are as follows: (1) the original signal equals the sum of all the IMFs, and (2) the sum of the modal bandwidths is the least. Moreover, a Lagrange multiplier is introduced as follows:

$$
L\left(\left\{u_{k}\right\},\left\{\omega_{k}\right\}, \lambda\right)=\alpha \sum_{k}\left\|\partial_{t}\left[\left(\delta(t)+\frac{j}{\pi t}\right) * u_{k}(t)\right] e^{-j \omega_{k} t}\right\|_{2}^{2}+\left\|f(t)-\sum_{k} u_{k}(t)\right\|_{2}^{2}+\left\langle\lambda(t), f(t)-\sum_{k} u_{k}(t)\right\rangle,
$$


where $\alpha$ denotes a penalty factor, guaranteeing the decomposition precision, and $\lambda$ is a Lagrangian multiplier to assure the rigidity of the constraint conditions. The optimal solution to the above optimization problem is achieved as follows:

$$
\begin{aligned}
\widehat{u}_{k}^{n+1}(\omega) & =\frac{\hat{f}(\omega)-\sum_{i=1}^{k-1} \hat{u}_{i}^{n+1}(\omega)-\sum_{i=k+1}^{K} \hat{u}_{i}^{n}(\omega)+\left(\hat{\lambda}^{n}(\omega) / 2\right)}{1+2 \alpha\left(\omega-\omega_{k}^{n}\right)^{2}}, \\
\omega_{k}^{n+1} & =\frac{\int_{0}^{\infty} \omega\left|\hat{u}_{k}(\omega)\right|^{2} \mathrm{~d} \omega}{\int_{0}^{\infty}\left|\hat{u}_{k}(\omega)\right|^{2} \mathrm{~d} \omega},
\end{aligned}
$$

where $u_{k}(t)$ is an IMF, $\widehat{u}_{k}^{n+1}(\omega)$ is the Fourier transform of $u_{k}(t)$, and $n$ denotes the number of iterations to resolve the problem.

2.2. Binary Genetic Algorithm. The BGA algorithm [48] is a novel evolutionary computation approach. BGA-based wrapper approach can be used for feature reduction. Compared with other metaheuristic algorithms, BGA shows superior learning capability and is more efficient to obtain a subset of relevant features. BGA can be described as follows: (1) initialize the chromosome population and complete binary encoding for each chromosome; (2) perform the crossover process and the mutation process to eliminate the weak chromosome. (3) If the exit condition is satisfied, return the subset of features that has optimally fitted chromosomes. The whole process is shown in Algorithm 1 , where $m$ is the population's size and $t_{\max }$ is the number of iterations.

2.3. Least Squares Support Vector Regression. The LSSVM [49] method is a simplified variant of SVM based on equality constraints. Assume a dataset $\{x, y\}, i=1,2, \ldots, N$, and the equation of regression can be defined as follows:

$$
y=W^{T} \Phi(x)+b,
$$

where $W$ is the weight vector, $\Phi(x)$ is the kernel function, and $b$ is the bias term. $W$ can be obtained by minimizing the following objective function:

$$
C=\frac{1}{2} W^{T} W+\frac{1}{2} \gamma \sum_{i=1}^{N} e_{i}^{2},
$$

subject to

$$
W^{T} \Phi(x)+b=1-e_{i},
$$

where $e_{i}$ is the error and $\gamma$ is the constant of regulation. A Lagrange function is introduced to resolve the above function as follows:

$$
L(W, b, e, \alpha)=\frac{1}{2}\|W\|^{2}+\gamma \sum_{i=1}^{N} e_{i}^{2}-\sum_{i=1}^{N} \alpha_{i}\left(W^{T} \Phi(x)+b-y_{i}+e_{i}\right) .
$$

Finally, the solution is achieved as follows:

$$
y=\sum_{i=1}^{N} \alpha_{i} \Phi\left(x_{i}\right) \Phi(x)+b,
$$

where $\alpha_{i}$ are Lagrange multipliers.

2.4. Echo State Network. The ESN [50] is a recurrent neural network. There are three layers in an ESN, including an input layer, a dynamic reservoir layer, and an output layer. The hidden neurons can be fully connected in the dynamic reservoir layer, and this layer can keep untrained during the network updates. The output layer is proposed to achieve the prediction by combining the outputs of the reservoirs. During the network updates, the internal states can be calculated as follows:

$$
\mathbf{x}_{\mathbf{t}+1}=\mathbf{f}\left(\mathbf{W}_{\mathrm{in}} \mathbf{v}_{\mathbf{t}+1}+\mathbf{W} \mathbf{x}_{\mathbf{t}}\right),
$$

where $\mathbf{x}_{\mathbf{t}+1}$ denotes the states in time $t+1, \mathbf{f}(\cdot)$ represents the activation function, $\mathbf{W}_{\text {in }}$ denotes the input layer coefficients, $\mathbf{v}_{\mathbf{t}+1}$ denotes the input vector, $\mathbf{W}$ represents the reservoir matrix, and $\mathbf{x}_{\mathbf{t}}$ denotes the states in time $t$. Besides, the reservoir weight matrix $\mathbf{W}$ can be adopted randomly. Finally, the prediction output $\mathbf{y}_{\mathbf{t}+1}$ can be obtained as follows:

$$
\mathbf{y}_{\mathbf{t}+1}=\mathbf{W}_{\text {out }} \mathbf{x}_{\mathbf{t}+1} \text {, }
$$

where $\mathbf{W}_{\text {out }}$ denotes the synaptic weights of the output layer, which can be adjusted by Moore-Penrose pseudo-inverse.

2.5. Online Sequential Extreme Learning Machine. The ELM is a novel feedforward network with a single-hidden layer. The mathematical expression of ELM is illustrated as follows:

$$
f(x)=G(x) \beta,
$$

where $G(x)$ is the hidden layer output matrix and $\beta$ is the output weight vector between the single-hidden layer to the output layer. The optimal solution of $\beta$ can be obtained as

$$
\beta^{*}=G^{\dagger} T,
$$

where $G^{\dagger}$ is the Moore-Penrose inverse of $G$ and $\mathbf{T}$ is the training-target matrix.

OSELM is a novel online learning algorithm [51]. The algorithm can be divided into two phases: the initialization phase and the online learning phase. In the initialization phase, given a training dataset $T$, the hidden layer output matrix $N_{0}$ and the output weight vector $\beta_{0}^{*}$ can be calculated as follows:

$$
\begin{aligned}
N_{0} & =\left(G_{0}^{T} G_{0}\right)^{-1}, \\
\beta_{0}^{*} & =N_{0} G_{0}^{T} T .
\end{aligned}
$$

Then, the online learning process starts, and the algorithm learns the data block by block. In the $\mathrm{k}_{\mathrm{th}}$ iteration, given a batch of new observed training-target matrix $T_{0}$. The output weight vector $\beta_{k}^{*}$ can be calculated as follows: 


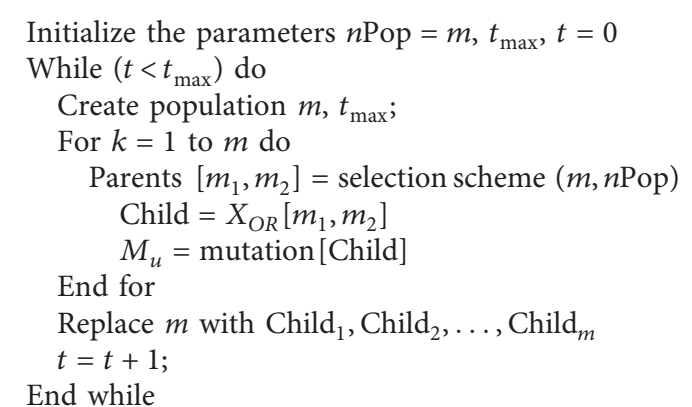

Return the optimal features which have the highest fitness value

Algorithm 1: Binary genetic algorithm.

$$
\begin{aligned}
& N_{k}=N_{k-1}-\frac{N_{k-1} G_{k} G_{k}^{T} N_{k-1}}{1+G_{k}^{T} N_{k-1} G_{k}}, \\
& \beta_{k}^{*}=\beta_{k-1}^{*}+N_{k} G_{k}\left(T_{0}-G_{k}^{T} \beta_{k-1}^{*}\right) .
\end{aligned}
$$

2.6. Bare-Bones Fireworks Algorithm. The BBFWA [52] is proposed as a simple, efficient, and powerful variant of the fireworks algorithm (FA). In FA, firework sparks are generated as candidate solutions, and the firework position is adopted as the optimal solution. In the process of BBFWA, there is one firework and several sparks. In an iteration, the exploration and exploitation are balanced by the size of search space, in which new solutions are generated. If the current optimal solution is improved, the size of the search space is reduced. Contrarily, if the current best solution is enhanced, the size of the search space is increased. The whole process of BBFWA is shown in Algorithm 2 . In the algorithm, $L_{b}$ and $U_{b}$ denote the search space's boundaries, $x$ represents the current best solution, and $f(x)$ indicates an objective function. Initially, solutions are sampled uniformly in a hyperrectangle $[x-A, x+A]$. If the best solution is not improved, parameter $A$ is multiplied by constant $C>1$. Alternatively, parameter $\mathrm{A}$ is multiplied by constant $C<1$.

In this paper, BBFWA is used to find the optimal parameters for the hybrid models, including the VMD-BGABBFWA-OSELM model, the VMD-BGA-BBFWA-LSSVR model, and the VMD-BGA-BBFWA-ESN model. For each model, the objective function of BBFWA is defined as the prediction result of 3 -fold cross-validation for a hybrid model. The input variables of the objective function are the hyperparameters of the hybrid model. The output variable of the objective function is the mean absolute percent error of 3-fold cross-validation. The objective function is defined as follows:

$$
f_{\text {obj }}=\log \left(1+\mathrm{CV}_{3}\right)
$$

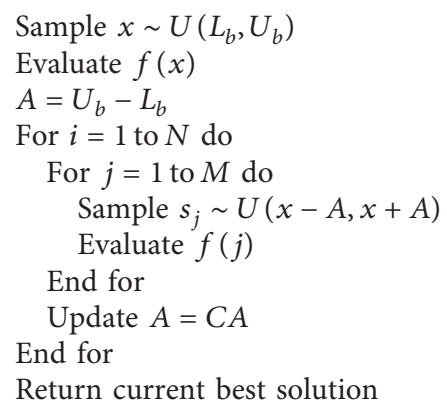

Return current best solution

Algorithm 2: Bare-bones fireworks algorithm.

where $\mathrm{CV}_{3}$ denotes the 3 -fold cross-validation loss on the training dataset.

2.7. Partial Least Squares Regression. In this paper, partial least squares regression (PLSR) [53] is used to reduce the prediction error from BO-LSTM. Given a linear model $y=\alpha+X \beta+\epsilon$, where $X$ is the explanatory variable, $y$ is the response, $\alpha$ and $\beta$ are the regression parameters, and $\epsilon$ is the error term. The process of PLSR can be explained in Algorithm 3 as follows.

2.8. The Framework of the Proposed Model. In this section, the proposed model, referred to as VMD-BGA-BBFWA-Ens, is presented. The framework and the flowchart of the proposed model are demonstrated in Figures 1 and 2. The modelling process of the proposed method is described as follows:

(1) Step 1: VMD is applied to decompose the original wind time series into several sublayers.

(2) Step 2: BGA is employed to reserve critical features from the sublayers of VMD. BGA is used to determine the most relevant features from the past twenty data points of the wind speed. 


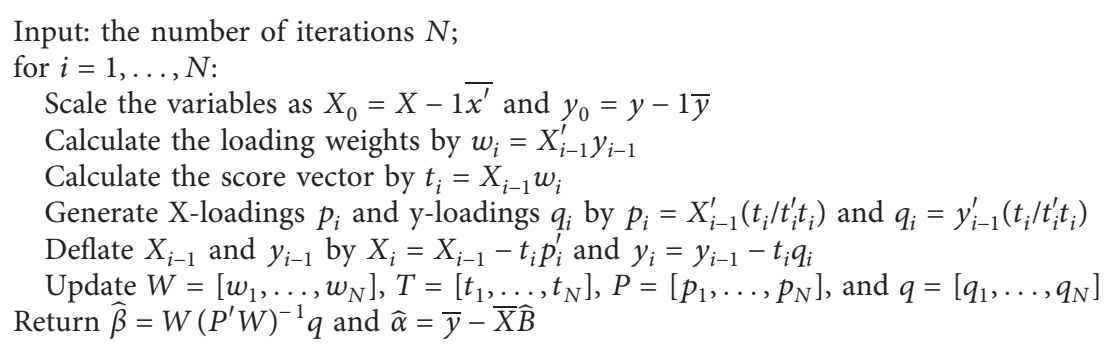

Algorithm 3: The partial least squares regression.

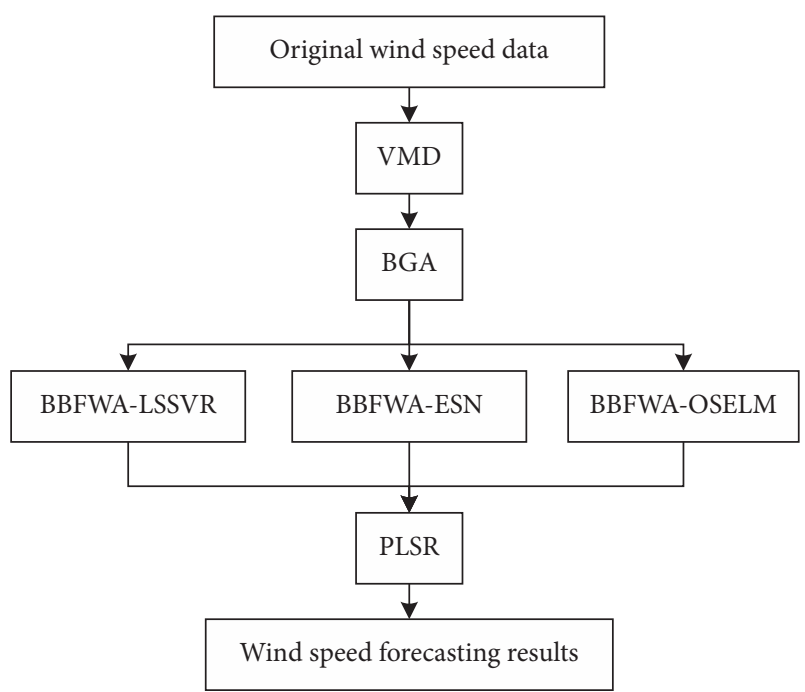

FIgURE 1: The framework of the proposed model.

(3) Step 3: three single prediction models, namely, the LSSVR model, the ESN model, and the OSELM model, are adopted to complete the forecasting for the VMD decomposition components independently. For each model, all the sublayers are predicted separately. The prediction results of each sublayer are aggregated to obtain the prediction values for the model. Moreover, the hyperparameters of each model are adjusted by BBFA.

(4) Step 4: to improve each model's forecasting accuracy and stability, PLSR is utilized to calculate the three forecasting models' weights and combine all prediction results from each model to generate the final prediction results.

(5) Step 5: several evaluation criteria are used to verify the proposed model. The proposed model is evaluated and compared with ten comparison models, including the LSSVR model, the ESN model, the OSELM model, the BGA-LSSVR model, the EMD-BGA-LSSVR model, the EEMD-BGA-LSSVR model, the VMD-BGA-LSSVR model, the VMD-BGA-BBFWA-ESN model, the VMD-BGA-BBFWA-LSSVR model, and the VMDBGA-BBFWA-OSELM model.

\section{Experimental Simulation}

This section demonstrates the details of the prediction process. Besides, comparison models are introduced to prove the validity of the proposed model.

3.1. Datasets. In this paper, two wind speed time series are used to evaluate the proposed model. These data were collected by the National Renewable Energy Laboratory (NREL) in 2012. The descriptive statistics of the data are given in Table 2. Each dataset contains 1800 points with $10 \mathrm{~min}$ interval. Each original dataset is divided into a training dataset and a test dataset. The training dataset includes 1-1700 points, and the test dataset contains 1701-1800 points. The wind time series is depicted in Figures 3 and 4, respectively.

3.2. Evaluation Metrics. In this paper, the performance of the involved models can be evaluated by the mean absolute error (MAE), the mean absolute percent error (MAPE), and the root mean square error (RMSE). The smaller the evaluation metrics, the better the model performed. The MAE, MAPE, and RMSE are defined as

$$
\begin{aligned}
\text { MAE } & =\frac{1}{N} \sum_{i=1}^{N}\left|\hat{y}_{i}-y_{i}\right|, \\
\text { MAPE } & =\frac{1}{N} \sum_{i=1}^{N} \frac{\left|\hat{y}_{i}-y_{i}\right|}{y_{i}} \times 100 \%, \\
\text { RMSE } & =\sqrt{\frac{1}{N} \sum_{i=1}^{N}\left(\hat{y}_{i}-y_{i}\right)^{2},}
\end{aligned}
$$

where $\hat{y}_{i}$ and $y_{i}$ denote the predicted and observed values at the time $i$, respectively, and $N$ represents the number of data points. Besides, improved percentage indices $P_{\mathrm{MAE}}, P_{\mathrm{MAPE}}$, and $P_{\text {RMSE }}$ are used to compare two models' performance. The $P_{\mathrm{MAE}}, P_{\mathrm{MAPE}}$, and $P_{\mathrm{RMSE}}$ are defined as 


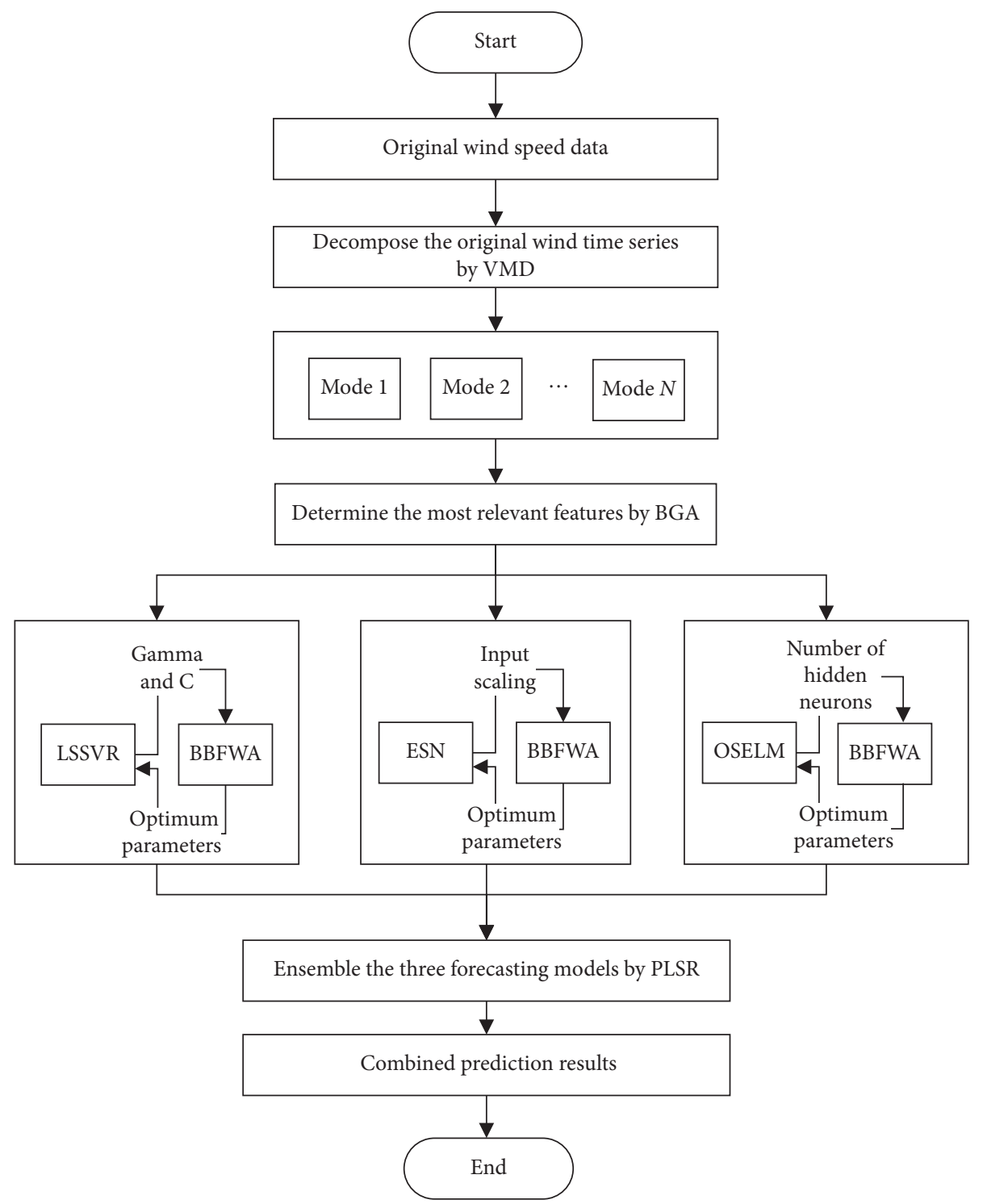

Figure 2: Flowchart of the proposed VMD-BGA-BBFWA-Ens model.

TABLe 2: Descriptive statistics of the wind speed datasets.

\begin{tabular}{lcccccc}
\hline Wind series & Min & Max & Mean & Variance & Skewness & Kurtosis \\
\hline 1 & 0.0 & 15.76 & 3.71 & 6.73 & 1.47 & 2.89 \\
2 & 0.0 & 22.76 & 5.46 & 14.24 & 1.22 & 1.27 \\
\hline
\end{tabular}

$$
\begin{gathered}
P_{\mathrm{MAE}}=\left(\frac{\mathrm{MAE} 1-\mathrm{MAE} 2}{\mathrm{MAE} 1}\right) \times 100, \\
P_{\mathrm{MAPE}}=\left(\frac{\mathrm{MAPE} 1-\mathrm{MAPE} 2}{\mathrm{MAPE} 1}\right) \times 100, \\
P_{\mathrm{RMSE}}=\left(\frac{\mathrm{RMSE} 1-\mathrm{RMSE} 2}{\mathrm{RMSE} 1}\right) \times 100,
\end{gathered}
$$

where MAE1, MAPE1, and RMSE1 denote the corresponding MAE, MAPE, and RMSE values of one model and
MAE2, MAPE2, and RMSE2 are the corresponding MAE, MAPE, and RMSE values of another model.

3.3. Pearson's Test. Pearson's test can evaluate the prediction capability of the involved models. In Pearson's test, the correlation coefficient is calculated to describe the degree of association between the observed data and the predicted data. If the correlation coefficient is 0 , then the practical and the predicted values are not correlated. If the coefficient is 1 , the observed and the predicted values are $100 \%$ correlated. 


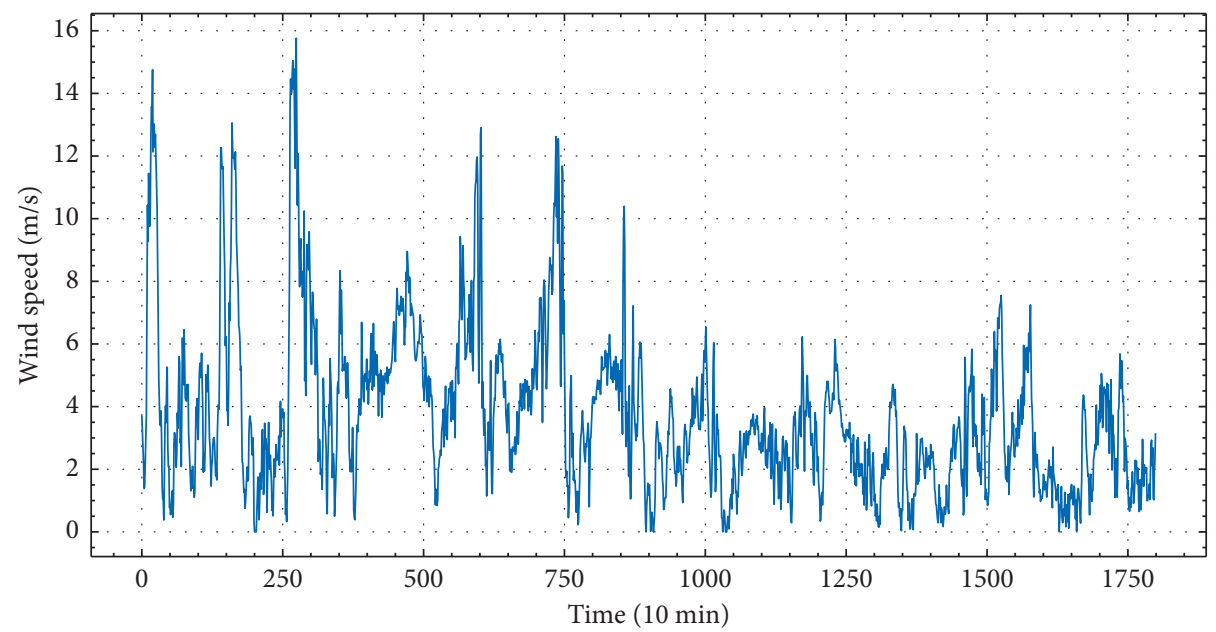

FIgURE 3: Original wind speed series 1.

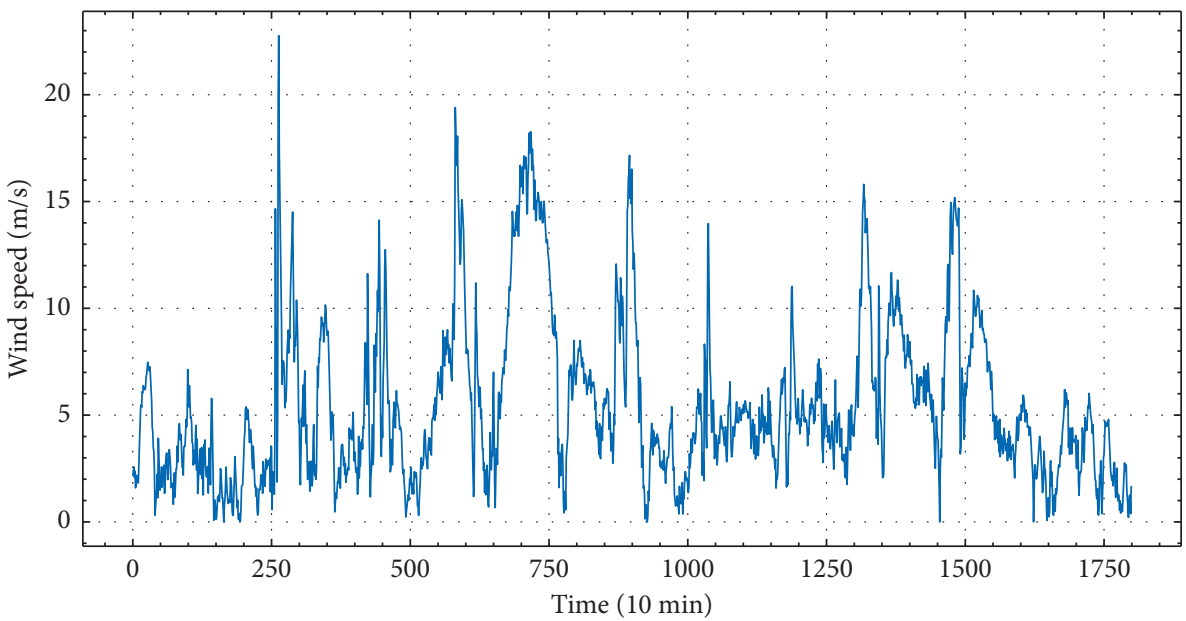

Figure 4: Original wind speed series 2.

The larger the Pearson's correlation coefficient is, the better the model is. Pearson's correlation coefficient can be described as follows:

$$
P=\frac{\sum_{i=1}^{N}\left(Y_{i}-Y_{i}\right)\left(\hat{Y}_{i}-\hat{Y}_{m}\right)}{\sqrt{\sum_{i=1}^{N}\left(Y_{i}-Y_{m}\right)^{2} \times \sum_{i=1}^{N}\left(\hat{Y}_{i}-\hat{Y}_{m}\right)^{2}}},
$$

where $Y_{i}$ is the actual data; $\widehat{Y}_{i}$ is the forecasting data; $Y_{m}$ and $\widehat{Y}_{m}$ are the means of the actual data and the forecasting data, respectively; and $N$ denotes the number of data points.

3.4. Results of Feature Selection. In this section, the chromosome of BGA is denoted as $X=\left[x_{1}, x_{2}, \ldots, x_{i}, \ldots, x_{10}\right]^{T}$, where $x_{i} \in\{0,1\}$ is the gen. If $x_{i}=1$, the feature $i$ is selected; conversely, if $x_{i}=0$, the feature $i$ is reduced. In the feature selection process, the number of population is 20 , the number of max iteration is 500 , and the probability of mutation is 0.05 . The results of the feature selection are listed in Table 3. It is seen that some IMFs are deleted from the feature set. For instance, in wind series 2, only 7 IMFs will be fed into the prediction models, and therefore, the conciseness of the proposed model is improved.

3.5. Parameter Selection. To demonstrate the proposed model's performance, four single models and six hybrid models are implemented, including the ESN model, the LSSVR model, the OSELM model, the BGA-LSSVR model, the EMD-BGA-LSSVR model, the EEMD-BGA-LSSVR model, the VMD-BGA-LSSVR model, the VMD-BGABBFWA-ESN model, the VMD-BGA-BBFWA-LSSVR model, and the VMD-BGA-BBFWA-OSELM model.

The default model parameters are determined using the trial-and-error approach. In the ESN models, the size of the reservoir is 500, and the input scaling is 0.5 . In the LSSVR models, the kernel function is RBF, the gamma is 0.01 , and the $\mathrm{C}$ is 400 . In the OSELM models, the number of hidden 
TABLE 3: The results of the feature selection of the wind speed time series.

\begin{tabular}{lcccccccccccc}
\hline Wind series & IMF 1 & IMF 2 & IMF 3 & IMF 4 & IMF 5 & IMF 6 & IMF 7 & IMF 8 & IMF 9 & IMF 10 \\
\hline 1 & 1 & 1 & 1 & 1 & 1 & 1 & 0 & 1 & 1 & 0 \\
2 & 1 & 1 & 1 & 1 & 0 & 1 & 0 & 0 & 1 & 1 \\
\hline
\end{tabular}

neurons is 10. The number of EMD trials is adopted as 100 . The number of EEMD trials is adopted as 100, and the standard deviation of Gaussian noise is 0.05 . The number of VMD modes for decomposition is 10 .

The optimal parameters of hybrid models are selected using the BBFWA algorithm. In the VMD-BGA-BBFWAOSELM model, the number of hidden neurons of OSELM is determined by BBFWA, and the search range is as $[10,200]$. In the VMD-BGA-BBFWA-LSSVR model, the gamma and $\mathrm{C}$ are optimized, the search space of gamma is as $[0.01,1]$, and the search space of $C$ is as $[100,1000]$. In the VMDBGA-BBFWA-ESN model, the input scaling of ESN is optimized, and the search range is as $[0.01,1]$. In BBFWA, the number of sparks is 10 , the number of iterations is 200 , and the fitness function is defined as the mean absolute percent error of 3 -fold cross-validation on the training dataset. In the optimization, the fitness drops as the iteration increases. Finally, the optimal parameters are obtained after 200 iterations, which are listed in Table 4.

3.6. Experimental Results. In this section, the forecasting results for wind speed series 1 and 2 are depicted in Figures 5-8. The estimation prediction results for wind speed series 1 and 2 are presented in Tables 5 and 6 . The improving percentages of the comparison models by the proposed model for wind speed series 1 and 2 are shown in Tables 7 and 8. Pearson's test results for wind speed series 1 and 2 are given in Tables 9 and 10.

\section{Discussion}

4.1. Comparison of the Decomposition Algorithms. This section analyzes the effect of wind speed data preprocessing. Three representative signal decomposition algorithms are compared. From Tables 5 and 6, it is seen as follows:

(1) Signal decomposition is always practical to enhance wind prediction accuracy. For instance, in wind series 1, the MAE values of the BGA-LSSVR model, the EMD-BGA-LSSVR model, the EEMD-BGALSSVR model, and the VMD-BGA-LSSVR model are $0.64 \mathrm{~m} / \mathrm{s}, \quad 0.45 \mathrm{~m} / \mathrm{s}, \quad 0.38 \mathrm{~m} / \mathrm{s}, \quad$ and $\quad 0.22 \mathrm{~m} / \mathrm{s}$, respectively.

(2) Moreover, the experimental results indicate that EEMD outperforms EMD in improving the prediction accuracy; for instance, in wind series 2, the MAPE values of the EMD-BGA-LSSVR model and the EEMD-BGA-LSSVR model are $28.81 \%$ and $24.18 \%$, respectively. The reason is that EMD has the endpoint effect and mode mixing problem, and EEMD can resolve these defects.
(3) However, EEMD is sensitive to white noise. To overcome this shortcoming, VMD is proposed. Table 6 indicates that $\mathrm{VMD}$ is more robust to data noise than EEMD. For example, in wind series 2, the RMSE values of the EEMD-BGA-LSSVR model and the VMD-BGA-LSSVR model are $0.38 \mathrm{~m} / \mathrm{s}$ and $0.34 \mathrm{~m} / \mathrm{s}$, respectively.

From the above analysis, it is concluded that VMD performs best, and it is reasonable to apply it in our proposed model.

4.2. Comparison of the Model Performances. This section discusses the prediction accuracy of the involved models. From Tables 5-10, it can be found as follows:

(1) The accuracy of the involved models is distinctly different but acceptable. It indicates that intelligent models are feasible for wind speed forecasting. However, the precision depends on models.

(2) The accuracy of the single models is low; for instance, in wind series 1, the MAPE values of the ESN model, the LSSVR model, and the OSELM model are $30.60 \%, 35.01 \%$, and $31.55 \%$, respectively. The reasons are that (a) original wind speed series has redundant information, (b) wind speed series has inherent prediction complexity, and (c) parameters of the models are not optimal.

(3) The accuracy of the single models can be improved by feature selection; for example, in wind series 1 , the MAPE values of the LSSVR model and the BGALSSVR model are $35.01 \%$ and $31.13 \%$, respectively. With feature selection, the input dimension is reduced, and the models achieve more robustness to data perturbation.

(4) To further reduce the prediction complexity, signal decomposition is introduced. Section 4.1 has analyzed the effect of different decomposition algorithms. The analysis shows that VMD performs best; for example, in wind series 2, the MAPE values of the EMD-BGA-LSSVR model, the EEMD-BGA-LSSVR model, and the VMD-BGA-LSSVR model are $28.81 \%, 24.18 \%$, and $21.04 \%$, respectively.

(5) Moreover, to enhance the involved models' structures, we use BBFWA to optimize the model parameters. The experimental results indicate that BBFWA can significantly reduce prediction errors. For instance, in wind series 1, the MAPE values of the VMD-BGA-LSSVR model and the VMD-BGABBFWA-LSSVR model are $21.04 \%$ and $12.03 \%$, respectively. 
TABLE 4: Optimal parameters of the prediction models.

\begin{tabular}{lccc}
\hline Wind series & Model & Parameter & Value \\
\hline & ESN & Input scaling & 0.01 \\
1 & LSSVR & Gamma, C & $0.01,994$ \\
& OSELM & Number of hidden neurons & 52 \\
\hline & ESN & Input scaling & 0.02 \\
2 & LSSVR & Gamma, C & $0.01,337$ \\
& OSELM & Number of hidden neurons & 20 \\
\hline
\end{tabular}

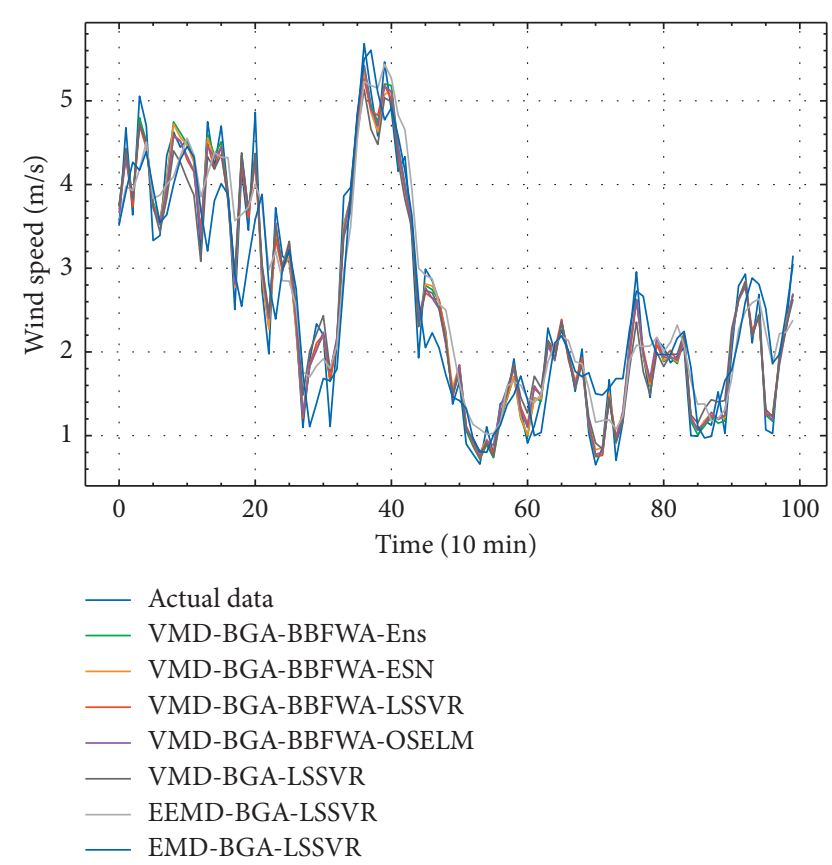

FIGURE 5: The forecasting results of hybrid models for wind speed series 1 .

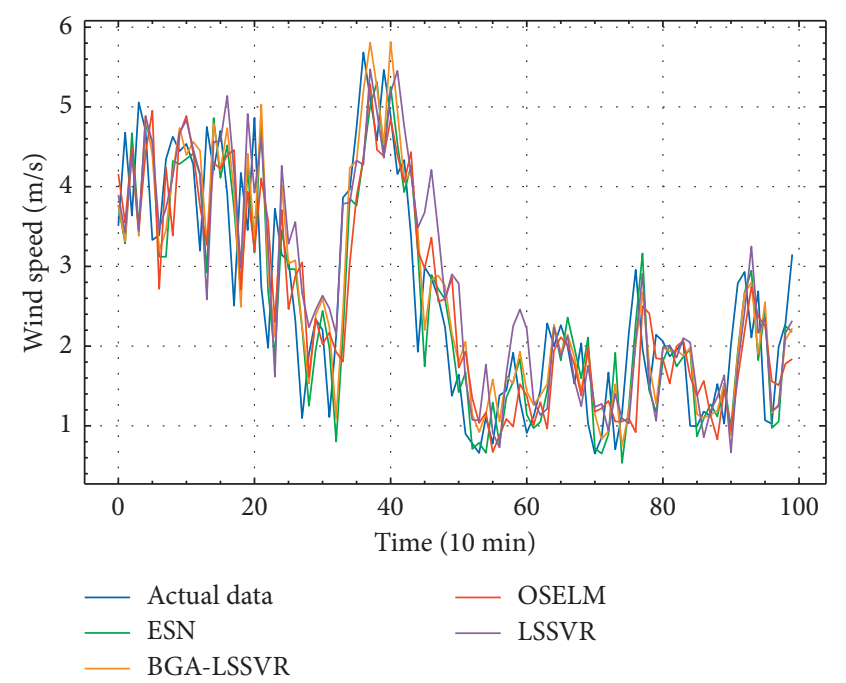

FIGURE 6: The forecasting results of single models for wind speed series 1 .

(6) However, each model has inherent limitations. In this paper, an ensemble model is proposed to overcome the defects of individual models. Tables 7 and 8 indicate that the proposed model significantly outperforms the single models; for instance, in wind series 1 , the MAE value is reduced by $74.27 \%$ from the ESN model to the proposed model. Besides, the proposed model also outperforms the hybrid models; for instance, in wind series 2, from the EMD-BGALSSVR model to the proposed model, the RMSE value is reduced by $62.85 \%$; from the EEMD-BGALSSVR model to the proposed mode, the RMSE value is decreased by $52.98 \%$.

(7) Finally, Pearson's test is conducted to verify the capability of the proposed model. Tables 9 and 10 demonstrate that Pearson's coefficient of the proposed model is significantly higher than that of the other models.

4.3. Comparison of the Model Efficiency. Besides prediction accuracy, efficiency is essential to evaluate model capability. From Tables 5 and 6, several findings are seen as follows:

(1) Single models are the most efficient; for example, in both wind series, the execution time of the ESN model, the LSSVR model, and the OSELM model is less than $10 \mathrm{~s}$. However, their prediction accuracy is not satisfactory; for instance, in wind series 1 , the MAPE values of the single models are always higher than $30 \%$.

(2) To further enhance the accuracy of the single models, feature selection is introduced. The experimental results indicate that BGA increases the execution time of the models; for instance, in wind series 1 , the running time of the LSSVR model and the BGALSSVR model is $6.97 \mathrm{~s}$ and $7.53 \mathrm{~s}$, respectively. It reveals that the execution time of feature selection is acceptable. Therefore, BGA is feasible to wind speed forecasting.

(3) Moreover, to reduce the prediction complexity, data preprocessing is employed. In wind series 1 , the execution time of the BGA-LSSVR model, the EMDBGA-LSSVR model, the EEMD-BGA-LSSVR model, and the VMD-BGA-LSSVR model is $7.53 \mathrm{~s}, 12.08 \mathrm{~s}$, $12.11 \mathrm{~s}$, and $11.33 \mathrm{~s}$, respectively. It indicates that signal decomposition algorithms may require more execution time than the feature selection method.

(4) Besides, BBFWA is employed for model parameter tuning. Compared with feature selection and data preprocessing, BBFWA significantly increases the 


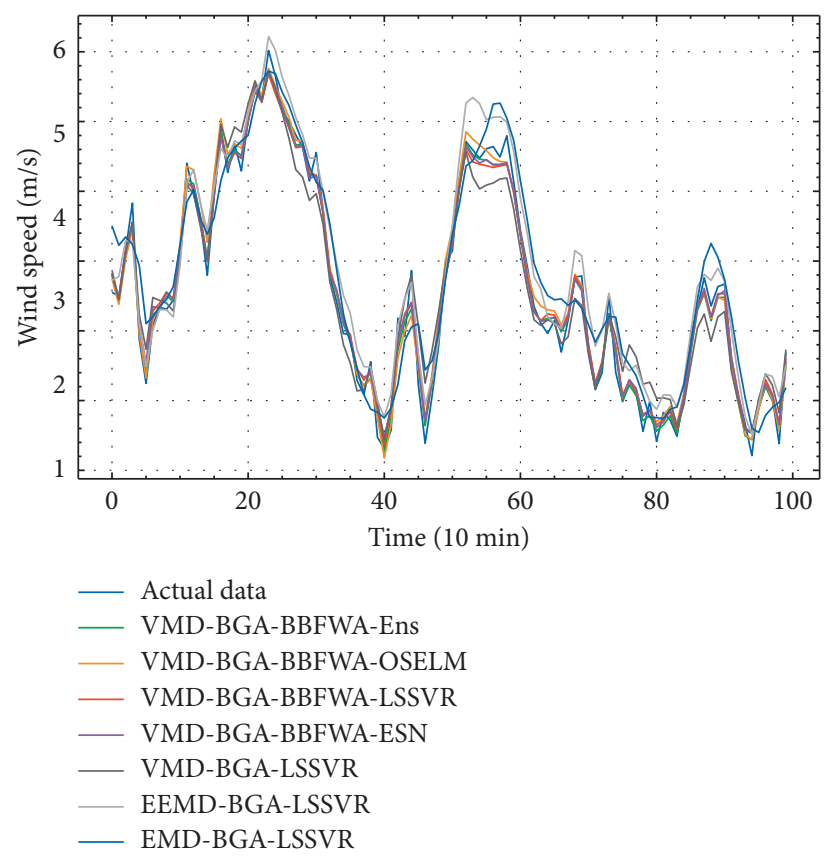

FiguRE 7: The forecasting results of hybrid models for wind speed series 2 .

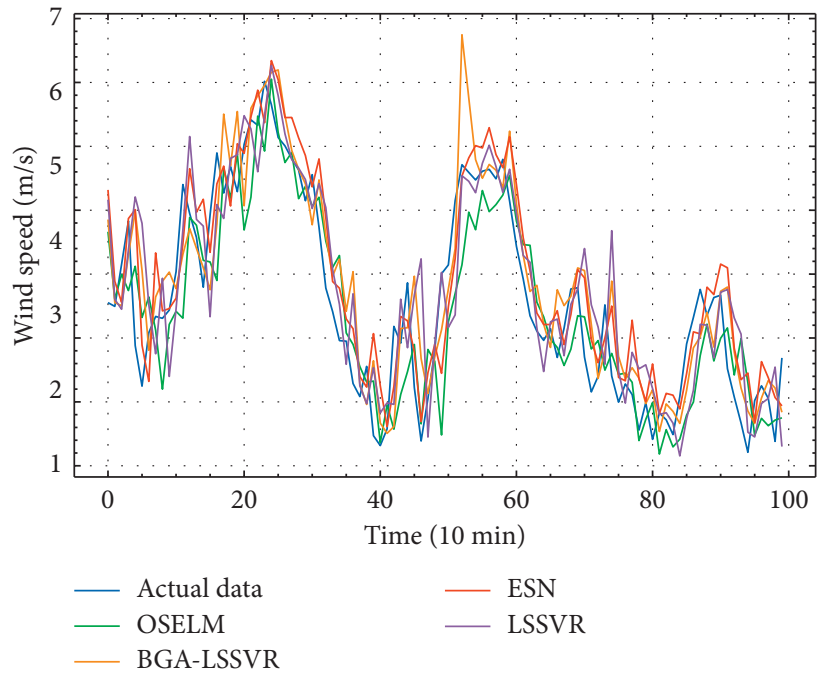

FIGURE 8: The forecasting results of single models for wind speed series 2 .

TABLE 5: Estimation prediction result for wind speed series 1 .

\begin{tabular}{|c|c|c|c|c|}
\hline Model & $\operatorname{MAE}(\mathrm{m} / \mathrm{s})$ & MAPE (\%) & RMSE $(\mathrm{m} / \mathrm{s})$ & Time (s) \\
\hline VMD-BGA-BBFWA-Ens & 0.17 & 7.97 & 0.20 & 80.27 \\
\hline VMD-BGA-BBFWA-ESN & 0.17 & 8.54 & 0.21 & 46.68 \\
\hline VMD-BGA-BBFWA-LSSVR & 0.18 & 8.91 & 0.23 & 30.36 \\
\hline VMD-BGA-BBFWA-OSELM & 0.18 & 9.15 & 0.22 & 3.21 \\
\hline VMD-BGA-LSSVR & 0.22 & 11.02 & 0.27 & 11.33 \\
\hline EEMD-BGA-LSSVR & 0.38 & 20.34 & 0.47 & 12.11 \\
\hline EMD-BGA-LSSVR & 0.45 & 24.11 & 0.58 & 12.08 \\
\hline ESN & 0.65 & 30.60 & 0.82 & 0.62 \\
\hline BGA-LSSVR & 0.64 & 31.13 & 0.80 & 7.53 \\
\hline OSELM & 0.65 & 31.55 & 0.84 & 0.01 \\
\hline LSSVR & 0.70 & 35.01 & 0.88 & 6.97 \\
\hline
\end{tabular}


TABLE 6: Estimation prediction result for wind speed series 2.

\begin{tabular}{|c|c|c|c|c|}
\hline Model & $\operatorname{MAE}(\mathrm{m} / \mathrm{s})$ & MAPE (\%) & RMSE $(\mathrm{m} / \mathrm{s})$ & Time $(s)$ \\
\hline VMD-BGA-BBFWA-Ens & 0.14 & 9.99 & 0.18 & 79.81 \\
\hline VMD-BGA-BBFWA-OSELM & 0.17 & 11.83 & 0.21 & 2.89 \\
\hline VMD-BGA-BBFWA-LSSVR & 0.15 & 12.03 & 0.19 & 30.71 \\
\hline VMD-BGA-BBFWA-ESN & 0.15 & 12.33 & 0.19 & 47.02 \\
\hline VMD-BGA-LSSVR & 0.28 & 21.04 & 0.34 & 12.01 \\
\hline EEMD-BGA-LSSVR & 0.32 & 24.18 & 0.38 & 12.97 \\
\hline EMD-BGA-LSSVR & 0.40 & 28.81 & 0.47 & 10.62 \\
\hline OSELM & 0.65 & 39.35 & 0.81 & 0.01 \\
\hline BGA-LSSVR & 0.63 & 43.31 & 0.80 & 8.08 \\
\hline ESN & 0.60 & 44.82 & 0.73 & 0.60 \\
\hline LSSVR & 0.70 & 52.66 & 0.90 & 7.04 \\
\hline
\end{tabular}

TABLE 7: Improving percentages of the comparison models by the proposed model for wind speed series 1 .

\begin{tabular}{|c|c|c|c|}
\hline Model & $\mathrm{P}_{\mathrm{MAE}}(\%)$ & $\mathrm{P}_{\text {MAPE }}(\%)$ & $\mathrm{P}_{\text {RMSE }}(\%)$ \\
\hline VMD-BGA-BBFWA-Ens vs. VMD-BGA-BBFWA-ESN & 2.73 & 6.69 & 3.65 \\
\hline VMD-BGA-BBFWA-Ens vs. VMD-BGA-BBFWA-LSSVR & 8.81 & 10.54 & 10.25 \\
\hline VMD-BGA-BBFWA-Ens vs. VMD-BGA-BBFWA-OSELM & 9.56 & 12.87 & 8.94 \\
\hline VMD-BGA-BBFWA-Ens vs. VMD-BGA-LSSVR & 24.60 & 27.63 & 25.19 \\
\hline VMD-BGA-BBFWA-Ens vs. EEMD-BGA-LSSVR & 55.82 & 60.81 & 56.96 \\
\hline VMD-BGA-BBFWA-Ens vs. EMD-BGA-LSSVR & 63.15 & 66.93 & 65.16 \\
\hline VMD-BGA-BBFWA-Ens vs. ESN & 74.27 & 73.94 & 75.21 \\
\hline VMD-BGA-BBFWA-Ens vs. BGA-LSSVR & 74.02 & 74.39 & 74.65 \\
\hline VMD-BGA-BBFWA-Ens vs. OSELM & 74.37 & 74.73 & 76.03 \\
\hline VMD-BGA-BBFWA-Ens vs. LSSVR & 76.20 & 77.23 & 76.92 \\
\hline
\end{tabular}

TABLE 8: Improving percentages of the comparison models by the proposed model for wind speed series 2 .

\begin{tabular}{|c|c|c|c|}
\hline Model & $\mathrm{P}_{\mathrm{MAE}}(\%)$ & $\mathrm{P}_{\text {MAPE }}(\%)$ & $\mathrm{P}_{\text {RMSE }}(\%)$ \\
\hline VMD-BGA-BBFWA-Ens vs. VMD-BGA-BBFWA-OSELM & 15.30 & 15.45 & 15.81 \\
\hline VMD-BGA-BBFWA-Ens vs. VMD-BGA-BBFWA-LSSVR & 7.91 & 16.84 & 7.81 \\
\hline VMD-BGA-BBFWA-Ens vs. VMD-BGA-BBFWA-ESN & 6.50 & 18.85 & 6.25 \\
\hline VMD-BGA-BBFWA-Ens vs. VMD-BGA-LSSVR & 48.33 & 52.44 & 47.90 \\
\hline VMD-BGA-BBFWA-Ens vs. EEMD-BGA-LSSVR & 55.59 & 58.63 & 52.98 \\
\hline VMD-BGA-BBFWA-Ens vs. EMD-BGA-LSSVR & 64.12 & 65.28 & 62.85 \\
\hline VMD-BGA-BBFWA-Ens vs. OSELM & 78.21 & 74.57 & 78.16 \\
\hline VMD-BGA-BBFWA-Ens vs. BGA-LSSVR & 77.35 & 76.90 & 77.86 \\
\hline VMD-BGA-BBFWA-Ens vs. ESN & 76.19 & 77.68 & 75.72 \\
\hline VMD-BGA-BBFWA-Ens vs. LSSVR & 79.60 & 81.00 & 80.46 \\
\hline
\end{tabular}

TABle 9: The results of Pearson's test for wind speed series 1.

\begin{tabular}{lc}
\hline Model & Pearson's coefficient \\
\hline OSELM & 0.7941 \\
LSSVR & 0.7965 \\
ESN & 0.8150 \\
BGA-LSSVR & 0.8252 \\
EMD-BGA-LSSVR & 0.9037 \\
EEMD-BGA-LSSVR & 0.9404 \\
VMD-BGA-LSSVR & 0.9837 \\
VMD-BGA-BBFWA-LSSVR & 0.9874 \\
VMD-BGA-BBFWA-OSELM & 0.9876 \\
VMD-BGA-BBFWA-ESN & 0.9889 \\
VMD-BGA-BBFWA-Ens & 0.9891 \\
\hline
\end{tabular}

TABLE 10: The results of Pearson's test for wind speed series 2.

\begin{tabular}{lc}
\hline Model & Pearson's coefficient \\
\hline LSSVR & 0.8269 \\
OSELM & 0.8556 \\
BGA-LSSVR & 0.8780 \\
ESN & 0.9050 \\
EMD-BGA-LSSVR & 0.9581 \\
EEMD-BGA-LSSVR & 0.9774 \\
VMD-BGA-LSSVR & 0.9854 \\
VMD-BGA-BBFWA-OSELM & 0.9908 \\
VMD-BGA-BBFWA-ESN & 0.9936 \\
VMD-BGA-BBFWA-LSSVR & 0.9935 \\
VMD-BGA-BBFWA-Ens & 0.9937 \\
\hline
\end{tabular}


execution time of the models. For instance, in wind series 2, the execution time of the BGA-LSSVR model, the VMD-BGA-LSSVR model, and the VMD-BGA-BBFWA-LSSVR model is $8.08 \mathrm{~s}, 12.01 \mathrm{~s}$, and $30.71 \mathrm{~s}$, respectively.

(5) Finally, the proposed model is the least efficient, while it achieves the best accuracy. The reason is that the proposed model ensembles several individual models, and it has the highest computational complexity. For instance, in wind series 2, the execution time of the VMD-BGA-BBFWA-ESN model, the VMD-BGA-BBFWA-LSSVR model, the VMDBGA-BBFWA-OSELM model, and the proposed model is $47.02 \mathrm{~s}, 30.71 \mathrm{~s}, 2.89 \mathrm{~s}$, and $79.81 \mathrm{~s}$, respectively.

In summary, the above analysis indicates that prediction accuracy and efficiency are contradictory. Ensemble models may achieve better accuracy; however, they lead to lower efficiency. It will be interesting to study how to improve the efficiency of ensemble models in future research.

4.4. Computational Complexity Analysis. In Section 4.3, the forecasting efficiency is presented based on the experimental results. To further investigate the efficiency of the proposed model, computational complexity analysis is conducted. First, the time complexity of BBFWA is presented, and then, the time complexity of the proposed model is discussed.

Assume that the big $\mathrm{O}$ notation denotes an algorithm's time complexity, $f(x)$ indicates the objective function, $P$ represents the number of iterations of BBFWA, $Q$ means the number of sparks, and $O(f)$ denotes time complexity of the objective function. From Section 2.6, the time complexity of the BBFWA can be estimated as follows:

$$
O(\text { BBFWA })=P * Q * O(f)
$$

The above formula indicates that the time complexity of BBFWA is linear with the number of sparks. Therefore, it is one of the most efficient optimization methods considering the order.

The time complexity of the proposed model is discussed as follows. Given that $N$ represents the number of training samples, the time complexity of OSELM is $O(N)$, and the computational complexity of LSSVR is $O\left(N^{2}\right)$. Given that $N_{x}$ denotes the number of reservoir neurons, $N_{u}$ is the size of the input, and the computational complexity of ESN is $O\left(\left(N_{x}+N_{u}+1\right)^{2} N\right)$. Since BBFWA is linear, the time complexity of the proposed ensemble model can be approximately estimated as follows:

$$
\begin{aligned}
O(\text { proposed model })= & P * Q *\left(O(N)+O\left(N^{2}\right)\right. \\
& \left.+O\left(\left(N_{x}+N_{u}+1\right)^{2} N\right)\right)
\end{aligned}
$$

In summary, the above discussion concludes that the proposed model's time complexity depends primarily on the parameters of BBFWA and the number of training samples.
TABLE 11: Estimation prediction result for various VMD mode numbers.

\begin{tabular}{lcccc}
\hline Number of modes & $\begin{array}{c}\text { MAE } \\
(\mathrm{m} / \mathrm{s})\end{array}$ & MAPE (\%) & RMSE (m/s) & Time (s) \\
\hline 4 & 0.38 & 19.71 & 0.49 & 192.41 \\
5 & 0.33 & 17.81 & 0.42 & 210.59 \\
6 & 0.32 & 16.48 & 0.40 & 260.05 \\
7 & 0.25 & 12.93 & 0.31 & 341.23 \\
8 & 0.21 & 10.17 & 0.26 & 343.71 \\
9 & 0.19 & 8.92 & 0.23 & 376.77 \\
10 & 0.17 & 7.97 & 0.20 & 481.64 \\
\hline
\end{tabular}

4.5. Sensitivity Analysis. The proposed model relies on the number of decomposition modes of VMD, which has to be preconfigured. In this section, several cases are conducted to discuss the sensitivity of the number of modes. The proposed model has performed predictions for the wind time series 1 with the various numbers of modes. The forecasting results are shown in Table 11. From Table 11, it is concluded that the prediction errors of the proposed model can be reduced when the number of decomposition modes increases; for instance, when the number of modes increases from 4 to 5 , the MAE index is improved by $13.16 \%$; when the number of modes increases from 7 to 8 , the MAE index is enhanced by $16.00 \%$, the MAPE index is reduced by $21.35 \%$, and the RMSE index is decreased by $16.13 \%$.

\section{Conclusion}

With environmental pollution becoming severe, the installed capacity of wind energy has increased over the years. Wind speed prediction is of great significance to improve the utilization of wind energy. However, the wind speed is highly random, and individual forecasting models cannot achieve satisfactory accuracy and robustness. Thus, in this paper, an ensemble model for short-term wind speed prediction was proposed based on the variational mode decomposition, three nonlinear regression models, and the no negative constraint theory. The binary genetic algorithm was used for feature selection, and the bare-bones fireworks algorithm was employed to improve the prediction accuracy. The model performance was evaluated on two datasets. The conclusions are as follows: (1) the decomposition algorithm of VMD can effectively decrease the prediction errors. The experimental results reveal that the VMD-based models can be superior to other comparison models. (2) The BBFWA algorithm can be suitable for improving the prediction performance; for example, the VMD-BGA-BBFWA-LSSVR model has a lower MAPE value than the VMD-BGA-LSSVR model, with the MAPE values of $8.91 \%$ and $12.03 \%$. (3) The proposed ensemble approach can obtain the best forecasting accuracy than other comparison models; for instance, the MAPE values of the proposed model are $7.97 \%$ and $9.99 \%$. (4) Pearson's test indicates that there is a significant difference between the proposed model and other involved models. In conclusion, the proposed method fully utilizes the virtues of VMD, BBFWA, and ensemble learning, and it is suitable for the forecasting of short-term wind speed. 
Future research directions will focus on improving the ensemble framework for multistep wind speed prediction.

\section{Data Availability}

The data that support the findings of this study are available from the corresponding author upon reasonable request.

\section{Conflicts of Interest}

The authors declare that they have no conflicts of interest.

\section{Acknowledgments}

This research was fully supported by the National Natural Science Foundation of China (Grant no. 51308553).

\section{References}

[1] M. Santhosh, C. Venkaiah, and D. M. Vinod Kumar, "Current advances and approaches in wind speed and wind power forecasting for improved renewable energy integration: a review," English Reports, vol. 2, no. 6, 2020.

[2] H. Liu and C. Chen, "Data processing strategies in wind energy forecasting models and applications: a comprehensive review," Applied Energy, vol. 249, pp. 392-408, 2019.

[3] X. Zhao, J. Liu, D. Yu, and J. Chang, "One-day-ahead probabilistic wind speed forecast based on optimized numerical weather prediction data," Energy Conversion and Management, vol. 164, pp. 560-569, 2018.

[4] J. B. Olson, "Improving wind energy forecasting through numerical weather prediction model development," Bulletin of the American Meteorological Society, vol. 100, no. 11, 2019.

[5] X. Liu, J. Zhou, H. Jia, Y. Mu, T. Wang, and C. Dai, "Correction method of wind speed prediction based on nonparametric kernel density estimation and numerical weather prediction," Dianli Zidonghua ShebeiElectric Power Autom. Equip.vol. 37, no. 10, 2017.

[6] Y. Zhang, Y. Zhao, C. Kong, and B. Chen, "A new prediction method based on VMD-PRBF-ARMA-E model considering wind speed characteristic," Energy Conversion and Management, vol. 203, Article ID 112254, 2020.

[7] O. Karaku, E. E. Kuruolu, and M. A. Altinkaya, "One-day ahead wind speed/power prediction based on polynomial autoregressive model," IET Renewable Power Generation, vol. 11, no. 11, 2017.

[8] S. Qin, J. Wang, J. Wu, and G. Zhao, "A hybrid model based on smooth transition periodic autoregressive and Elman artificial neural network for wind speed forecasting of the Hebei region in China," International Journal of Green Energy, vol. 13, no. 6, 2016.

[9] A. A. Kadhem, N. I. A. Wahab, I. Aris, J. Jasni, and A. N. Abdalla, "Advanced wind speed prediction model based on a combination of Weibull distribution and an artificial neural network," Energies, vol. 10, no. 11, 2017.

[10] J. Wang, C. Wu, and T. Niu, "A novel system for wind speed forecasting based on multi-objective optimization and echo state network," Sustainability, vol. 11, no. 2, 2019.

[11] Z. Tian, G. Wang, S. Li, Y. Wang, and X. Wang, "Artificial bee colony algorithmoptimized error minimized extreme learning machine and its application in short-term wind speed prediction," Wind Engineering, vol. 43, no. 3, 2019.
[12] D. Zhang, X. Peng, K. Pan, and Y. Liu, "A novel wind speed forecasting based on hybrid decomposition and online sequential outlier robust extreme learning machine," Energy Conversion and Management, vol. 180, pp. 338-357, 2019.

[13] R. Li and Y. Jin, "A wind speed interval prediction system based on multi-objective optimization for machine learning method," Applied Energy, vol. 228, pp. 2207-2220, 2018.

[14] C. Fu, G.-Q. Li, K.-P. Lin, and H.-J. Zhang, "Short-term wind power prediction based on improved chicken algorithm optimization support vector machine," Sustainability, vol. 11, no. 2, 2019.

[15] H. Ren, X. Lei, and P. Zhang, "A study of wind speed prediction based on particle swarm algorithm to optimize the parameters of sparse least squares support vector," International Journal of. Simulation. Systems, Science \& Technology, vol. 17, no. 30, 2016.

[16] N. Bokde, A. Feijóo, and K. Kulat, "Analysis of differencing and decomposition preprocessing methods for wind speed prediction," Applied Soft Computing, vol. 71, pp. 926-938, 2018.

[17] N. Bokde, A. Feijoo, D. Villanueva, and K. Kulat, “A review on hybrid empirical mode decomposition models for wind speed and wind power prediction," Energies, vol. 12, no. 2, 2019.

[18] R. Ye, Z. Guo, R. Liu, and J. Liu, "Short-term wind speed forecasting method based on wavelet packet decomposition and improved elman neural network," in Proceedings of the 2016 International Conference on Probabilistic Methods Applied to Power Systems (PMAPS), Beijing, China, December 2016.

[19] X. Mi, H. Liu, and Y. Li, "Wind speed prediction model using singular spectrum analysis, empirical mode decomposition and convolutional support vector machine," Energy Conversion and Management, vol. 180, pp. 196-205, 2019.

[20] Y. Yang and Y. Yang, "Hybrid prediction method for wind speed combining ensemble empirical mode decomposition and bayesian ridge regression," IEEE Access, vol. 8, pp. 71206-71218, 2020.

[21] M. Santhosh, C. Venkaiah, and D. M. V. Kumar, "Short-term wind speed forecasting approach using ensemble empirical mode decomposition and deep Boltzmann machine," Sustainable Energy, Grids and Networks, vol. 19, pp. 80-95, 2019.

[22] Y. Huang, L. Yang, S. Liu, and G. Wang, "Multi-step wind speed forecasting based on ensemble empirical mode decomposition, long short term memory network and error correction strategy," Energies, vol. 12, no. 10, 2019.

[23] P. Jiang, R. Li, and H. Li, "Multi-objective algorithm for the design of prediction intervals for wind power forecasting model," Applied Mathematical Modelling, vol. 67, pp. 101-122, 2019.

[24] X. Wang, Q. Yu, and Y. Yang, "Short-term wind speed forecasting using variational mode decomposition and support vector regression," Journal of Intelligent \& Fuzzy Systems, vol. 34, no. 6, pp. 3811-3820, 2018.

[25] Q. Wu and H. Lin, "Short-term wind speed forecasting based on hybrid variational mode decomposition and least squares support vector machine optimized by bat algorithm model," Sustainability, vol. 11, no. 3, 2019.

[26] S. Sun, L. Wei, J. Xu, and Z. Jin, "A new wind speed forecasting modeling strategy using two-stage decomposition, feature selection and Dawnn," Energies, vol. 12, no. 3, 2019.

[27] G. Memarzadeh and F. Keynia, "A new short-term wind speed forecasting method based on fine-tuned LSTM neural network and optimal input sets," Energy Conversion and Management, vol. 213, 2020. 
[28] N. Huang, E. Xing, G. Cai, Z. Yu, B. Qi, and L. Lin, "Shortterm wind speed forecasting based on low redundancy feature selection," Energies, vol. 11, no. 7, p. 1638, 2018.

[29] Y. K. Semero, J. Zhang, D. Zheng, and D. Wei, “An accurate very short-term electric load forecasting model with binary genetic algorithm based feature selection for microgrid applications," Electric Power Components and Systems, vol. 46, no. 14-15, 2018.

[30] H. Liu, Z. Duan, H. Wu, Y. Li, and S. Dong, "Wind speed forecasting models based on data decomposition, feature selection and group method of data handling network," Measurement, vol. 148, Article ID 106971, 2019.

[31] H. Liu, H. Tian, X. Liang, and Y. Li, "New wind speed forecasting approaches using fast ensemble empirical model decomposition, genetic algorithm, Mind Evolutionary Algorithm and Artificial Neural Networks," Renewable Energy, vol. 83, pp. 1066-1075, 2015.

[32] E. C. Bezerra, R. P. S. Leao, and A. P. Braga, “A self-adaptive approach for particle swarm optimization applied to wind speed forecasting," Journal of Control, Automation and Electrical Systems, vol. 28, no. 6, 2017.

[33] Y.-L. Hu and L. Chen, "A nonlinear hybrid wind speed forecasting model using LSTM network, hysteretic ELM and differential evolution algorithm," Energy Conversion and Management, vol. 173, pp. 123-142, 2018.

[34] S. P. H. Athar, D. Ziaei, and N. Goudarzi, "Artificial intelligence for optimal sitting of individual and networks of wind farms," in Proceedings of the American Society of Mechanical Engineers, Power Division (Publication) POWER, Salt Lake City, UT, USA, August 2019.

[35] C. Lei, B. Fang, H. Gao, W. Jia, and W. Pan, "Short-term power load forecasting based on least squares support vector machine optimized by bare bones fireworks algorithm," in Proceedings of 2018 IEEE 3rd Advanced Information Technology, Electronic and Automation Control Conference, IAEAC 2018, pp. 2231-2235, Chongqing, China, December 2018.

[36] Y. Ren, P. N. Suganthan, and N. Srikanth, "Ensemble methods for wind and solar power forecasting-a state-of-the-art review," Renewable and Sustainable Energy Reviews, vol. 50, pp. 82-91, 2015.

[37] L. Xiao, J. Wang, Y. Dong, and J. Wu, "Combined forecasting models for wind energy forecasting: a case study in China," Renewable and Sustainable Energy Reviews, vol. 44, pp. 271288, 2015.

[38] Z. Yang and J. Wang, "A combination forecasting approach applied in multistep wind speed forecasting based on a data processing strategy and an optimized artificial intelligence algorithm," Applied Energy, vol. 230, pp. 1108-1125, 2018.

[39] X. Niu and J. Wang, "A combined model based on data preprocessing strategy and multi-objective optimization algorithm for short-term wind speed forecasting," Applied Energy, vol. 241, pp. 519-539, 2019.

[40] L. Luo, H. Li, J. Wang, and J. Hu, "Design of a combined wind speed forecasting system based on decomposition-ensemble and multi-objective optimization approach," Applied Mathematical Modelling, vol. 89, pp. 49-72, 2021.

[41] P. Jiang and C. Li, "Research and application of an innovative combined model based on a modified optimization algorithm for wind speed forecasting," Measurement, vol. 124, pp. 395-412, 2018.

[42] Y. Chen, Z. Dong, Y. Wang et al., "Short-term wind speed predicting framework based on EEMD-GA-LSTM method under large scaled wind history," Energy Conversion and Management, vol. 227, Article ID 113559, 2021.

[43] J. Wang, Q. Li, and B. Zeng, "Multi-layer cooperative combined forecasting system for short-term wind speed forecasting," Sustainable Energy Technologies and Assessments, vol. 43, Article ID 100946, 2021.

[44] K. Dragomiretskiy and D. Zosso, "Variational mode decomposition," IEEE Transactions on Signal Processing, vol. 62, no. 3, pp. 531-544, 2014.

[45] S. Lahmiri, "Comparing variational and empirical mode decomposition in forecasting day-ahead energy prices," IEEE Systems Journal, vol. 11, no. 3, pp. 1907-1910, 2015.

[46] M. Zhang, Z. Jiang, and K. Feng, "Research on variational mode decomposition in rolling bearings fault diagnosis of the multistage centrifugal pump," Mechanical Systems and Signal Processing, vol. 93, pp. 460-493, 2017.

[47] J. Quan and L. Shang, "Short-term wind speed forecasting based on ensemble online sequential extreme learning machine and Bayesian optimization," Mathematical Problems in Engineering, vol. 2020, Article ID 7212368, 2020.

[48] E. Sopov, "Large-scale global optimization using a binary genetic algorithm with EDA-based decomposition," in Proceedings of the Lecture Notes in Computer Science (including subseries Lecture Notes in Artificial Intelligence and Lecture Notes in Bioinformatics), pp. 619-626, Bali, Indonesia, August 2016.

[49] X. Hong, R. Mitchell, and G. Di Fatta, "Simplex basis function based sparse least squares support vector regression," $\mathrm{Neu}$ rocomputing, vol. 330, pp. 394-402, 2019.

[50] Q. Wang, L. Wang, Y. Liu, J. Zhao, and W. Wang, "Time series prediction with incomplete dataset based on deep bidirectional echo state network," IEEE Access, vol. 7, pp. 152533152544, 2019.

[51] C. Chupong and B. Plangklang, "Comparison study on artificial neural network and online sequential extreme learning machine in regression problem," in Proceedings of the 20197 th International Electrical Engineering Congress (iEECON), Hua Hin, Thailand, March 2019.

[52] J. Li and Y. Tan, "The bare bones fireworks algorithm: a minimalist global optimizer," Applied Soft Computing, vol. 62, pp. 454-462, 2018.

[53] Q. Wang, Y. Lei, and H. Cao, "Wind power prediction based on nonlinear partial least square," Mathematical Problems in Engineering, vol. 2018, Article ID 6829274, 17 pages, 2018. 